\title{
On the Mathematical Constitution and Explanation of Physical Facts
}

\author{
Joseph Berkovitz ${ }^{1}$ \\ Forthcoming in M. Hemmo and O. Shenker (eds.), \\ Quantum, Probability, Logic: Itamar Pitowsky's Work and Influence
}

\begin{abstract}
The mathematical nature of modern physics suggests that mathematics is bound to play some role in explaining physical reality. Yet, there is an ongoing controversy about the prospects of mathematical explanations of physical facts and their nature. A common view has it that mathematics provides a rich and indispensable language for representing physical reality but that, ontologically, physical facts are not mathematical and, accordingly, mathematical facts cannot really explain physical facts. In what follows, I challenge this common view. I argue that, in addition to its representational role, in modern physics mathematics is constitutive of the physical. Granted the mathematical constitution of the physical, I propose an account of explanation in which mathematical frameworks, structures, and facts explain physical facts. In this account, mathematical explanations of physical facts are either species of physical explanations of physical facts in which the mathematical constitution of some physical facts in the explanans are highlighted, or simply explanations in which the mathematical constitution of physical facts are highlighted. In highlighting the mathematical constitution of physical facts, mathematical explanations of physical facts deepen and increase the scope of the understanding of the explained physical facts. I argue that, unlike other accounts of mathematical explanations of physical facts, the proposed account is not subject to the objection that mathematics only represents the physical facts that actually do the explanation. I conclude by briefly considering the implications that the mathematical constitution of the physical has for the question of the unreasonable effectiveness of the use of mathematics in physics.
\end{abstract}

\section{Outline}

1 The orthodoxy

2 An alternative perspective

3 On the relationship between mathematics and physics

4 On conceptions of mathematical constitution of the physical

5 On the common view of how mathematical models represent physical reality

6 On the notion of the physical

7 On the scope of the mathematical constitution of the physical

8 A sketch of a new account of mathematical explanation of physical facts

9 On mathematical explanations of physical facts

9.1 On a D-N explanation of the life cycle of 'periodical' cicadas

9.2 On structural explanation of the uncertainty relations

9.3 On abstract explanation of the impossibility of a minimal tour across the bridges of Königsberg

9.4 On explanations by constraints that are more necessary than laws of nature 10 Is the effectiveness of mathematics in physics unreasonable?

${ }^{1}$ Institute for the History and Philosophy of Science and Technology, University of Toronto, Victoria College, 91 Charles St West, Toronto, ON M5S 1K7, Canada. Email: joseph.berkovitz@utoronto.ca 


\section{The orthodoxy}

Modern physics is highly mathematical, and its enormous success seems to suggest that mathematics is very effective as a tool for representing the physical realm. Nobel Laureate Eugene Wigner famously expressed this view in "The unreasonable effectiveness of mathematics in the natural sciences":

the mathematical formulation of the physicist's often crude experience leads in an uncanny number of cases to an amazingly accurate description of a large class of phenomena (Wigner 1960, p. 8).

The great success of mathematical physics has led many to wonder about the causes of and reasons for the effectiveness of mathematics in representing the physical realm. Wigner thought that this success is unreasonable and even miraculous.

The miracle of the appropriateness of the language of mathematics for the formulation of the laws of physics is a wonderful gift which we neither understand nor deserve. (Ibid., 1960, p. 11)

Mathematics is commonly conceived as the study of purely abstract concepts and structures and the question is how such concepts and structures could be so successful in representing the physical realm. ${ }^{2}$

The ubiquity and great success of mathematics in physics does not only raise the puzzle of the "unreasonable" effectiveness of mathematics in physics. It also suggests that mathematics is bound to play a role in explaining physical reality. Yet, there is an ongoing controversy about the prospects and nature of mathematical explanations of physical facts. (Henceforth, the term 'physical fact' is meant to subsume all aspects of the physical, such as laws, principles, properties, relations, etc. Further, for the sake of

${ }^{2}$ Steiner $(1998$, p. 9) argues that there are two problems with Wigner's formulation of question of the "unreasonable" success of mathematics in the natural sciences. First, Wigner ignores the cases in which "scientists fail to find appropriate mathematical descriptions of natural phenomena" and the "mathematical concepts that never have found an application." Second, Wigner focuses on individual cases of successful applications of mathematical concepts, and these successes might have nothing to do with the fact that a mathematical concept was applied. Steiner seeks to formulate the question of the astonishing success of the applicability of mathematics in the natural sciences in a way that escapes these objections. He argues that in their discoveries of new theories, scientists relied on mathematical analogies. Often these analogies were 'Pythagorean', meaning that they were inexpressible in any other language but that of pure mathematics. That is, often the strategy that physicists pursued to guess the laws of nature was Pythagorean: "they used the relations between the structures and even the notations of mathematics to frame analogies and guess according to those analogies" (ibid., p. 4). Steiner argues that although not every guess, or even a large percentage of the guesses, was correct, this global strategy was astonishingly successful.

Steiner's reasoning and examples are intriguing and deserve an in-depth study, which, for want of space, I need to postpone for another opportunity. I believe, though, that such a study will not change the main thrust of my analysis of the question of the mathematical constitution of the physical and its implication for the questions of mathematical explanations of physical facts and the "unreasonable effectiveness of mathematics in the natural sciences". 
simplicity and continuity with the literature, I will use the term 'physical facts' to be more general so as to include natural facts.) A common view has it that mathematics provides a rich and indispensable language for representing physical reality but could not play a role in explaining physical facts. Another related prevalent view is that, ontologically, the physical is to be sharply distinguished from the mathematical. Thus, it is common to think that sharing mathematical properties does not entail sharing physical properties. The idea is that, fundamentally, physical facts are not mathematical, and that mathematics only provides a language for representing the physical realm, even if this language is indispensable in the sense of being by far the most effective. These common views about the nature of physical facts and the role that mathematics plays in physics seem to be dogmas of contemporary mainstream schools of philosophy of science. Accordingly, the idea that mathematical facts could explain physical facts seems puzzling: How could facts about abstract, non-physical entities, which obtain in all 'possible worlds' (including 'non-physical' ones) and are ontologically separated from the physical, explain physical facts?

It should not be surprising then that until recently the subject of mathematical explanations of physical facts drew little attention in the philosophical literature. Yet, a recent interest in philosophical questions concerning the application of mathematics in the natural sciences has led philosophers to study the prospects and nature of mathematical explanations of physical facts, and various accounts of such explanations have been proposed (see, for example, Steiner 1978, Clifton 1998, Colyvan 2001, 2002, Batterman 2002, 2010, 2018, Pincock 2004, 2011a,b, 2012, 2015, Baker 2005, 2009, 2017, Bokulich 2008a,b, 2011, Dorato and Felline 2011, Lyon 2012, Batterman and Rice 2014, Lange 2016, 2018, Baron, Colyvan and Ripley 2017, Bueno and French 2018, Felline 2018, Mancosu 2018). These accounts are clearly intended to offer non-causal explanations of physical facts. Yet, the exact nature of the explanations on offer is unclear. For example, some of these accounts attempt to show that mathematical facts or mathematical structures 'could make a difference' to physical facts (Bokulich 2008a,b, 2011, Pincock 2011b, 2015, Baron, Colyvan and Ripley 2017). But granted the common view that, ontologically, there is a sharp distinction between the physical and the mathematical, it is not clear how mathematical structures or mathematical facts could make such a difference and what the nature of the difference making is. (Hereafter, by 'mathematical facts' I will mean mathematical truths, such as $2+2=4$.)

Further, the term 'mathematical explanation of physical facts' is ambiguous. In what follows, by this term I shall mean explanations in which mathematical frameworks, structures, or facts explain physical facts. This kind of explanation is to be distinguished from explanations of physical facts that merely appeal to mathematical frameworks, structures, or facts in order to represent physical facts. In the literature, there are various putative examples of mathematical explanations of physical facts and attempts to subsume them under a general account. In Section 9, I will consider four such accounts. I will argue that the nature of explanation in these accounts is unclear and that they are subject to the objection that the mathematical frameworks, structures, or facts they appeal to play a representational rather than explanatory role. 


\section{An alternative perspective}

Although those who advocate the existence of mathematical explanations of physical facts endorse the idea of the indispensability of mathematics in physics, they seem to presuppose that mathematics does not play a constitutive role in defining what the physical is. I shall propose below that a way to circumvent the conundrum that current accounts of mathematical explanations of physical facts encounter is to acknowledge that, in modern natural sciences, the mathematical is constitutive of the physical. I believe that there are good reasons to think that contemporary natural science is in effect committed to such constitution. In particular, I will argue that if physical facts are fundamentally non-mathematical, the common wisdom about how mathematical models represent physical systems - which is based on the so-called 'mapping account' - becomes a mystery. Accordingly, the idea that there is a mathematical constitution of the physical should appeal to anybody who accepts the mapping account as a necessary part of mathematical representation of physical facts, independently of their view about whether mathematical explanations of physical facts exist. In fact, reflecting on the nature of the physical in contemporary physics, I will argue that this idea should also be compelling to those who reject the mapping account as a necessary part of mathematical representations.

Granted the mathematical constitution of the physical, I shall propose a new account of mathematical explanation of physical facts. The main idea of this account is that a mathematical explanation of physical facts highlights the mathematical constitution of some physical facts, thus deepening and increasing the scope of the understanding of these and other related physical facts. While this account could be applied to many of the putative examples of mathematical explanations of physical facts in the literature, the question whether it could account for all kinds of putative mathematical explanations of physical facts is beyond the scope of this paper for various reasons. First, as mentioned above, the term 'mathematical explanation of physical facts' is ambiguous and my understanding of it need not agree with all of its applications in the literature. Second, for lack of space and to keep things as simple as possible, I will only sketch the proposed account and consider it mainly in the context of simple examples of mathematical explanations of physical facts. Third, the range of putative examples I have encountered is not sufficiently broad to support such a grand claim. Yet, I think that there are good reasons to believe that the proposed account could be applied to all the examples of mathematical explanations of physical facts in which mathematical frameworks, structures, or facts are supposed to explain physical facts.

The current study was inspired by an exchange that I had with participants of a workshop on the role of mathematics in science at the Institute for the History and Philosophy of Science and Technology at the University of Toronto in October 2010. To my surprise, the common view was that while mathematics is very important for representing physical systems/states/properties/laws, it does not play any constitutive role in physics. Even more surprising, this view was also shared by supporters of ontic structural realism, for whom such a constitution seems to be a natural premise. The workshop on Mathematical and Geometrical Explanations at the Universitat Autònoma de Barcelona in March 2012 
and subsequent conferences and colloquia provided me with the opportunity to present the proposed account of mathematical explanation of physical facts, and the invitation to contribute to the second volume in honor of Itamar Pitowsky prompted me to write up this paper.

Pitowsky had a strong interest in mathematics and its use in physics. His research covers various topics in these fields: the philosophical foundations of probability; the nature of quantum probability and its relation to classical probability and quantum logic; the interpretation of quantum mechanics, in general, and as a theory of probability, in particular; quantum non-locality; computation complexity and quantum computation; and probability in statistical mechanics. This research has inspired a new perspective on the non-classical nature of quantum probability, quantum logic, and quantum non-locality, and influenced our understanding of these issues. ${ }^{3}$ Pitowsky's view of the relationship between mathematics and physics is less known, however. Based on his commentary on Lévy-Leblond's reflections on the use and effectiveness of mathematics in physics in the Bar-Hillel colloquium in the mid 1980s, in the next section I present his thoughts on these topics. Lévy-Leblond's and Pitowsky's reflections on the subject will provide a background for the main focus of the paper: the mathematical constitution and mathematical explanations of physical facts. Both Lévy-Leblond and Pitowsky embrace the idea of the mathematical constitution of the physical. Yet, it is not clear from their discussion what the exact nature of this constitution is. In Section 4, I review two traditional conceptions of mathematical constitution of physical facts - the Pythagorean and the neo-Kantian - and briefly give an example of mathematical constitution of physical facts. In Section 5, I review the common view of how mathematical models/theories represent physical facts. The main idea of this view is that a mathematical model/theory represents the properties of its target physical entities on the basis of a similarity between the relevant mathematical structure in the model/theory and the structure of the properties of these entities. More precisely, the idea is that there is an appropriate structure-preserving mapping between the mathematical system in the model/theory and the properties and relations of the represented physical entities. I will argue that the mapping account undermines the idea that physical facts are nonmathematical. Further, in Section 6, I will argue that a reflection on the concept of the physical in modern physics supports the idea that the physical is constituted by the mathematical. In Section 7, I will suggest that the philosophical interpretative frameworks of scientific theories/models in natural science determine the scope rather than the existence of the mathematical constitution of the physical. In Section 8, I will present the main ideas of the proposed account of mathematical explanation of physical facts. This account is sufficiently general so as to be integrated into the frameworks of various existing accounts of explanation. Accordingly, existing accounts of mathematical explanation of physical facts can be revised along the lines of the new account to yield accounts of explanations which embody the idea that mathematical explanations of physical facts highlight the mathematical constitution of physical facts and accordingly deepen and increase the scope of understanding of the explained physical facts. In Section 9, I will consider four existing accounts of mathematical explanation of physical

${ }^{3}$ For a list of Pitowsky's publications, see https://www.researchgate.net/scientificcontributions/72394418 Itamar Pitowsky. 
facts. I will argue that the exact nature of explanation in these accounts is unclear and, moreover, that they are subject to the objection that mathematics only plays a representational role, representing the physical facts that actually have the explanatory power. In Section 10, I will conclude by briefly commenting on the implications of the mathematical constitution of the physical for the question of the unreasonable effectiveness of mathematics in physics.

\section{On the relationship between mathematics and physics}

There is a broad consensus among physicists and philosophers that mathematics constitutes the language of modern physics. Poincaré expressed this view succinctly.

$[\mathrm{O}]$ rdinary language is too poor, it is also too vague, to express relationships so delicate, so rich, and so precise. This, then, is a primary reason why the physicist cannot do without mathematics; it furnishes him with the only language he can speak (Poincaré 1913, p. 141).

Lévy-Leblond (1992, p. 147) notes that the picture of mathematics as the language of physics is ambiguous and admits two different interpretations: mathematics as the intrinsic language of Nature, which has to be mastered in order to understand her workings; and mathematics as a special language into which facts of Nature need to be translated in order to be comprehended. He refers to Galileo and Einstein as advocates of the first view, and Heisenberg as an exponent of the second view. He does not think of these views as contradictory but rather as extreme positions on a continuous spectrum of opinions. Lévy-Leblond explores the question of the peculiar relation between mathematics and physics, and he contends that the view of mathematics as a special language of nature does not provide an answer to this question. Instead of explaining why mathematics applies so well to physics, this view is concerned with the supposed adequacy of mathematics as a language for acquiring knowledge of nature in general. In his view, in other natural sciences, such as chemistry, biology, and the earth sciences, we could talk about the relation between mathematics and the particular science as one of application, with the implication that "we are concerned with an instrumental relation, in which mathematics appears as a purely technical tool, external to its domain of implementation and independent of the concepts proper to that domain." By contrast, he argues that mathematics plays a much deeper role in physics and that the relation between mathematics and physics is not instrumental (ibid., p. 148). He maintains that mathematics is internalized by physics and, like Bachelard (1965), he characterizes the relation of mathematics to physics as that of "constitutivity" (ibid., p. 149).

Lévy-Leblond does not specify what he means by this relation. He notes, though, that by mathematics being constitutive of physics, he does not mean that a physical concept is to be identified with or reduced to its underlying mathematical concept(s). He also does not think of this relation as implying that a physical concept is a mathematical concept plus a physical interpretation: "the mathematical concept is not a skeleton fleshed out by physics, or an empty abstract form to be filled with concrete content by physics." And, 
again, following Bachelard, he does not conceive of the "distinction between a physical concept and its mathematical characterization" as static:

mathematics is a dynamical 'way of thinking', leading from experimental facts to the constitution of the physical concept (ibid., p. 149).

Further, Lévy-Leblond rejects the Platonic interpretation of the connection between mathematics and physics, according to which "the role of the physicist is to decipher Nature so as to reveal 'the hidden harmony of things' (Poincaré), as expressed by mathematical formulas, underlying the untidy complexity of physical facts." For him, the mathematical constitution of the physical does not imply "that every physical concept is the contingent materialization of an absolute mathematical being which is supposed to express its deepest truth, its ultimate essence" (ibid., p. 150). He takes the "mathematical polymorphism" of physics - namely, the possibility that physical laws and concepts may be endowed with several mathematizations - as evidence against the Platonic viewpoint and for the dynamical nature of the constitutive relation between mathematics and physics (ibid., pp. 150-152).

Lévy-Leblond also rejects the view that there is a preestablished harmony between physical and mathematical concepts. First, he draws attention to "the physical multivalence of mathematical structures (which is the converse of the mathematical polymorphism of physical laws), i.e., the possibility that the same mathematical theory may be adequate to several completely different physical domains" (ibid., p. 152). And, following Feynman et al. (1963, 2:12f), he maintains that "equations from different phenomena are so similar" not because of the physical identity of the represented systems, properties, or laws. Rather, it is due to "a common process of approximation/abstraction" that these phenomena are "encompassed by analogous mathematizations" (ibid., pp. 152-153). Second, he argues that, "contrary to some rash assertions", it is not true that the abstract constructions elaborated by mathematics are all useful for the physicist. He contends that, as mathematics develops on its own, the fraction of mathematical theories and ideas that are utilized by physics is decreasing rather rapidly: while the use of mathematics in physics keeps on growing, it does so much less rapidly than the development of mathematics that is independent of physics (ibid., $p$. 154).

Lévy-Leblond's reflections above are intended to probe the nature of the mathematical constitution of the physical and the special relationship between physics and mathematics. His main conclusions are that there is no pre-established harmony between physics and mathematics and that the singularity of physics in its relationship to mathematics is very difficult to account for on the view that mathematics is merely a language for representing the physical reality. Lévy-Leblond holds that if mathematics is conceived as merely a language, it "would necessarily have to be a universal one, applicable in principle to each and every natural science", and the specificity of physics "must then be treated as only a matter of degree, and criteria must be found to distinguish it from other sciences" (ibid., p. 156). He considers two possible ways to try to explain the specificity of physics as a matter of degree. One is the common view that physics is 
more advanced. The idea here is that "more precise experimental methods, a firmer control of experimental conditions, permit the quantitative measurement of physical notions, which are thus transformed into numerical magnitudes" (ibid., p. 157). The other explanation localizes

the specificity of physics in its objects of inquiry ... It is commonly stated that physics is "more fundamental" than the other natural sciences. Dealing with the deepest structures of the world, it aims to bring to light the most general laws of Nature, which are implicitly considered as the "simplest" and thus, in an Aristotelian way, as the "most mathematical" (ibid., p. 158).

Lévy-Leblond rejects both explanations. He contends that the first explanation per se fails to explain the privileged status of physics, and, moreover, it relies on a very naive view of mathematics. And he claims that the second explanation implies that "mathematicity assumes a normative character and becomes a criterion of scientificity", but the development of other sciences, such as chemistry, geology, and molecular biology seems to contradict this norm. He argues that these sciences are characterized by a clear-cut separation between their conceptual equipment and the mathematical techniques used - a separation that allows them to maintain a degree of autonomy even in the face of new developments in relevant branches of physics (ibid., pp. 148, 157-158).

In the end, Lévy-Leblond fails to find an explanation for the singular relationship between mathematics and physics. He thus opts for a radical solution according to which physics is defined as any area of the natural sciences where mathematics is constitutive (ibid., p. 158).

In his commentary on Lévy-Leblond's reflections, Pitowsky (1992, p. 166) finds LévyLeblond's characterization of physics very attractive. He points out, though, that it is incomplete, as the question arises as to what makes a given use of mathematics constitutive rather than a mere (non-constitutive) application. Indeed, as one could see from the above review, the questions of the nature of the mathematical constitution of the physical and the distinction between constitutive and non-constitutive uses of mathematics remain open. While Pitowsky shares Lévy-Leblond's intuition that the use of mathematics in physics is different and deeper, he finds it difficult to provide an analytical frame for this observation.

Pitowsky also addresses the question whether Lévy-Leblond's view of the relationship between mathematics and physics fits in with the reductionist ethos of science. He remarks that while "[o]ne of the central ideals in the scientific enterprise is to try and provide a unified explanation of all natural phenomena", the sharp lines of demarcation that Lévy-Leblond draws "between the sciences appears to obstruct this idea, which has had some remarkable manifestations" (ibid., pp. 166-167).

Further, Pitowsky considers Lévy-Leblond's observation that the ratio of mathematics to mathematical physics increases with time. While he finds this observation correct, he 
does not think that it settles the question of whether there is a harmony between mathematical structures and physical phenomena for two reasons (ibid., p. 163):

(a) It is impossible to foretell which portion of mathematics is relevant to physics and which is not. There is no clear a priori distinction between pure mathematics and mathematical physics.

(b) There is far more abstract mathematics involved in physics than one usually assumes.

To motivate these claims, Pitowsky discusses Bolzano's unintuitive example of a continuous function which is nowhere differentiable (ibid., pp. 163-165). The example was introduced at the beginning of the $19^{\text {th }} \mathrm{C}$, but was ignored for a few decades only to be resurrected and expanded by Weierstrass around the middle of the $19^{\text {th }} \mathrm{C} .{ }^{4}$ About 50 years later, Wiener (1923) showed, for the probability space of the set of all possible continuous trajectories of Brownian particles, that (with probability one) the path taken by a Brownian particle is nowhere differentiable. As Pitowsky notes, continuous nowhere differentiable functions and similar creatures also play a role in more recent theories as well, such as the theory of fractals (Mandelbrot 1977). This and other examples suggest that abstruse mathematics plays a subtle role in physical theories, which is not sufficiently appreciated, and that it is impossible to tell a priori whether any such piece of mathematics will figure in future theories of physics.

Pitowsky believes that such examples and the "entanglement of mathematical structure with physical fact" suggest that "there exists no a priori principle which distinguishes pure mathematics from applied mathematics, mathematical physics in particular", and that mathematical physics is much broader than it is commonly conceived. Thus, the question of the "correspondence" between mathematics and physics is unavoidable, and "one can understand it in a naive, realistic sense, or defend a thesis with a Kantian flavor" (ibid., pp. 165-166).

I find Lévy-Leblond's and Pitowsky's reflections on the relationship between mathematics and physics and the effectiveness of the use of mathematics in physics intriguing. I share with them the view that, in modern physics, mathematics is constitutive of the physical and that the use of mathematics in physics is more successful than in other natural sciences. I disagree, though, with Lévy-Leblond's opinion that mathematics is only constitutive in physics. I believe that mathematics is also constitutive in other natural sciences. Indeed, in both of the traditional frameworks of portraying the physical as constituted by the mathematical - the Pythagorean and the neo-Kantian - the constitution pertains to the physical broadly conceived across the natural sciences. Yet,

${ }^{4}$ Bolzano's example is believed to have been produced in the 1830 s but the manuscript was only discovered in 1922 (Kowalewski 1923) and published in 1930 (Bolzano 1930). Neuenschwander (1978) notes that Weierstrass presented a continuous nowhere function before the Royal Academy of Sciences in Berlin on 18 July 1872 and that it was published first, with his assent, by du Bois-Reymond in 1875 (du Bois-Reymond 1875) and later in Weierstrass (1895). 
having a unified framework does not entail that the natural sciences are reducible to physics.

I also share with Lévy-Leblond and Pitowsky the view that the question of the particular success of the use of mathematics in physics is very interesting, important, and open. Recall that Lévy-Leblond rejects two possible explanations for the specificity of physics among the natural sciences: one is that physics is more advanced, and the other is that the objects of physics are the simplest and most fundamental. I don't find his reasoning here compelling, and I believe that both of these lines of reasoning are worth pursuing. For example, Lévy-Leblond argues against the second explanation that it implies a false hierarchy of sciences where physics is the most scientific and the idea that all the natural sciences are reducible to physics. It is not clear to me, however, why the view that the objects of physics are the simplest and most fundamental has such implications. Indeed, one may maintain this view yet reject the suggested implications.

Finally, I share with Pitowsky the view that in modern physics there is a "correspondence" or "harmony" between the physical and the mathematical, which could be understood along Pythagorean or Kantian lines. I believe that such a correspondence/harmony can be accounted for by the mathematical constitution of the physical, and in the next section I will briefly review the nature of this constitution in the context of Pythagorean and neo-Kantian frameworks. I also agree with Pitowsky that Lévy-Leblond's observation that the ratio of mathematics to mathematical physics increases with time is not evidence against the correspondence/harmony between the physical and the mathematical, and, moreover, that there is no a priori distinction between pure and applied mathematics. And, similarly to him, I believe that a reflection on the history of science could support the absence of such a distinction.

The question of the specificity of physics among the natural sciences is very interesting and important and deserve an in-depth consideration. Yet, for want of space, I will have to postpone its discussion to another opportunity and focus on the constitutive role that mathematics plays in physics and its implications for mathematical explanations of physical facts and the question of the unreasonable effectiveness of the use of mathematics in physics. In Sections 5 and 6, I will argue that the idea of the mathematical constitution of the physical can be motivated by an analysis of how mathematical systems represent physical phenomena and reality. In Section 7, I will consider the relevance of the philosophical frameworks of theories/models in natural science for the mathematical constitution of the physical. Granted the mathematical constitution of the physical, in Section 8 I propose a new account of mathematical explanation of physical facts, in Section 9 I compare it to exiting accounts, and in Section 10 I briefly discuss the question of the unreasonable effectiveness of the use of mathematics in physics. But, first, I turn to briefly review two ways of thinking about the mathematical constitution of physical facts.

\section{On conceptions of mathematical constitution of the physical}

There are two traditional ways of conceiving the mathematical constitution of the physical. One is along what I shall call the 'Pythagorean picture'. There is no canonical 
depiction of this picture. In what follows, I will consider three versions of it, due to Aristotle, Galileo, and Einstein.

Aristotle describes the Pythagorean picture in the Metaphysics, Book 1, part 1.

[T]he so-called Pythagoreans, who were the first to take up mathematics, not only advanced this study, but also having been brought up in it they thought its principles were the principles of all things. Since of these principles numbers are by nature the first, and in numbers they seemed to see many resemblances to the things that exist and come into being - more than in fire and earth and water (such and such a modification of numbers being justice, another being soul and reason, another being opportunity - and similarly almost all other things being numerically expressible); since, again, they saw that the modifications and the ratios of the musical scales were expressible in numbers; since, then, all other things seemed in their whole nature to be modelled on numbers, and numbers seemed to be the first things in the whole of nature, they supposed the elements of numbers to be the elements of all things, and the whole heaven to be a musical scale and a number.

In The Assayer Galileo endorses a different version of the Pythagorean picture. In his characterization, the universe is written in the language of mathematics, the characters of which are geometrical figures.

Philosophy is written in this grand book - I mean the universe - which stands continually open to our gaze, but it cannot be understood unless one first learns to comprehend the language and interpret the characters in which it is written. It is written in the language of mathematics and its characters are triangles, circles, and other geometrical figures without which it is humanly impossible to understand a single word of it; without these, one is wandering a dark labyrinth.

In his lecture "On the Method of Theoretical Physics", Einstein also seems to endorse a version of the Pythagorean picture. He depicts nature as the realization of mathematical ideas.

Our experience hitherto justifies us in believing that nature is the realization of the simplest conceivable mathematical ideas. I am convinced that we can discover by means of purely mathematical constructions the concepts and the laws connecting them with each other, which furnish the key to the understanding of natural phenomena. (Einstein 1933/1954)

The Pythagorean picture has also been embraced by a number of other notable natural philosophers and physicists. While the exact nature of this picture varies from one version to another, all share the idea that mathematics constitutes all the elements of physical reality. Physical objects, properties, relations, facts, principles, and laws are mathematical by their very nature, so that ontologically one cannot separate the physical 
from the mathematical. This does not mean that physical things are numbers, as it may be tempting to interpret Aristotle's characterization in the Metaphysics and as it is frequently suggested. Rather, the idea is that mathematics defines the fundamental nature of physical quantities, relations, and structures. Without their mathematical properties and relations, physical things would have been essentially different from what they are. The Pythagoreans see the mathematical features of the physical as intrinsic to it, so that mathematics is not regarded merely as a 'language' or conceptual framework within which the physical is represented and studied.

The main challenge for the Pythagoreans is to identify the mathematical frameworks that define the nature of the physical, especially given that both mathematics and natural science continue to develop. The Pythagoreans may argue, though, that it is the role of mathematics and natural science to discover these frameworks.

The second traditional way of conceiving the mathematical constitution of the physical is along neo-Kantian lines, especially those of Herman Cohen and Ernest Cassirer of the Marburg school. Cassirer (1912/2005, p. 97) comments that Cohen held that

the most general, fundamental meaning of the concept of object itself, which even physiology presupposes, cannot be determined rigorously and securely except in the language of mathematical physics.

For example, in mechanics,

[w] hat motion "is" cannot be expressed except in concepts of quantity; understanding these presupposes a fundamental system of a pure doctrine of Quantity. Consequently, the principles and axioms of mathematics become the specific foundation that must be taken as fixed in order to give content and sense to any statement of natural science about actuality.

Cassirer held that mathematics is crucial for furnishing the fundamental scaffolding of physics, the intellectual work of understanding, and the construction of physical reality.

"Pure" experience, in the sense of a mere inductive collection of isolated observations, can never furnish the fundamental scaffolding of physics; for it is denied the power of giving mathematical form. The intellectual work of understanding, which connects the bare fact systematically with the totality of phenomena, only begins when the fact is represented and replaced by a mathematical symbol. (Cassirer 1910/1923, p. 147)

The idea is that "the chaos of impressions becomes a system of numbers" and "objective values" are obtained in "the transformation of impression into a mathematical "symbol'." The physical analysis of an object "into the totality of its numerically constants" is a judgment in which 
the concrete impression first changes into the physically determinate object. The sensuous quality of a thing becomes a physical object, when it is transformed into a serial determination. The "thing" now changes from a sum of properties into a mathematical system of values, which are established with reference to some scale of comparison. Each of the different physical concepts defines such a scale, and thereby renders possible an increasingly intimate connection and arrangement of the elements of the given. (Ibid., p. 149)

More generally, in Cassirer's view, the concepts of natural science are "products of constructive mathematical procedure." It is only when the values of physical constants "are inserted in the formulae of general laws, that the manifold of experiences gains that fixed and definite structure, that makes "nature'." The reality that natural science studies is a construction, and the construction is in mathematical terms.

The scientific construction of reality is only completed when there are found, along with the general causal equations, definite, empirically established, quantitative values for particular groups of processes: as, for example, when the general principle of the conservation of energy is supplemented by giving the fixed equivalence-numbers, in accordance with which the exchange of energy takes place between two different fields. (Ibid., p. 230)

In the neo-Kantian school, the mathematical constitution of natural science is the outcome of a historical process. The reality that mathematical natural science studies is a continuous serial process, and along this process the exact nature of the mathematics that constitutes the physical evolves.

A notable example of the mathematical constitution of the physical is the role that the calculus plays in shaping the concepts of modern physics. For example, the concept of the mathematical limit constitutes the concept of instantaneous velocity and more generally the concept of instantaneous change. This constitution marks a very significant change from the concepts of instantaneous velocity and instantaneous change before the calculus revolution. Consider, for instance, Zeno's arrow paradox. ${ }^{5}$ The paradox starts from the premise that time is composed of moments, which are indivisible, and that during any such moment the arrow has no time to move. Thus, since the arrow does not have time to move in any particular moment, it is concluded that the arrow can never be in motion. Zeno's paradox suggests that in the pre-calculus era instantaneous velocity does not make sense. By contrast, when, based on the calculus, instantaneous velocity is defined as the limit of a series of average velocities as the time interval approaches zero, Zeno's paradox can be circumvented. ${ }^{6}$

\footnotetext{
${ }^{5}$ For the arrow paradox and its analysis, see for example Huggett $(1999,2019)$ and references therein.

${ }^{6}$ Two comments: 1 . The introduction of the calculus is often presented as a solution to Zeno's arrow paradox. I believe that this presentation is somewhat misleading. The calculus does not really solve Zeno's paradox. It just evades it by fiat, i.e. by redefining the concept of instantaneous velocity. 2. For a recent example of the role that the calculus plays in constituting physical facts, see Stemeroff's (2018, Chapter 3) analysis of Norton's Dome - the thought
} 


\section{On the common view of how mathematical models represent physical reality}

In modern physics, the physical is characterized in mathematical terms. Yet, the common view is that, while mathematics provides a rich and indispensable language for representing the physical, the physical is fundamentally non-mathematical. If the physical is fundamentally non-mathematical, how could a mathematical model represent adequately physical systems?

The replies to this question are often based on a widespread view that the mathematical structures in the model are similar or identical to the structures of the physical things that the model represents. More precisely, the idea is that a mathematical model represents physical systems in terms of an appropriate structure-preserving mapping from the mathematical structures that the model posits to the structures of the physical things it represents. Chris Pincock (2004, 2011, 2012, chap. 2) calls this conception of representation the 'mapping account'.

For example, a model based on Newton's three laws of motion (together with the relevant 'boundary conditions') may represent the solar system. Newton's laws of motion are expressed in terms of mathematical relations.

First law: The net force $F$ on an object is zero just in case its velocity $v$ is constant:

$$
\sum F=0 \Leftrightarrow d v / d t=0
$$

where $t$ denotes time and $d v / d t$ denotes the derivative of velocity with respect to time.

Second law: The net force $F$ on an object is equal to the rate of change of its linear momentum $p, p=m v$, where $m$ denotes the object's mass:

$$
F=\frac{d p}{d t}=\frac{d(m v)}{d t}=m \frac{d v}{d t} .
$$

Third law: For every action there is an equal and opposite re-action. That is, for every force exerted by object $A, F_{A}$, on object $B, B$ exerts a reaction force, $F_{B}$, that is equal in size, but opposite in direction:

$$
F_{A}=-F_{B} .
$$

These mathematical relations are supposed to correspond to the physical relations that obtain between the forces acting on the planets and their masses, linear momenta, velocities, and accelerations, and the correspondence is achieved by a mathematical

experiment that is purported to show that non-deterministic behaviour could exist within the scope of Newtonian mechanics. Stemeroff argues that: (i) this thought experiment overlooks the constraints that the calculus imposes on Newton's theory; and that (ii) when these constraints are taken into account, Norton's Dome is ruled out as impossible in Newtonian universes. 
mapping from the model to the solar system.

In its simplest form, the mapping account posits that a mathematical model/theory represents physical systems because the mathematical structures it posits are isomorphic to the structures of properties of, and relations between these systems. Intuitively, the idea is that a mathematical model and properties and relations of the physical systems it represents share the same structures. More precisely, there is a one-to-one mapping between the mathematical structures in the model and properties of, and relations between the represented systems. Mathematical models/theories could also represent by means of weaker notions of structural identity, such as partial isomorphism (French and Ladyman 1999), embedding (Redhead 2001), or homomorphism (Mundy 1986). Some other accounts of how mathematical models/theories represent take the mapping account to be incomplete. In particular, Bueno and Colyvan (2011, p. 347) argue that, for the mapping account to get started, we need something like a pre-theoretic structure of the world. While it is common to think of mathematical structure as a set of objects and a set of relations on them (Resnik 1997, Shapiro 1997), Bueno and Colyvan remarks that "the world does not come equipped with a set of objects ... and sets of relations on those. These are either constructs of our theories of the world or identified by our theories of the world." Thus, the mapping account requires having what they call "an assumed structure" in order to get started (ibid.). Yet, Bueno and Colyvan accept the mapping account as a necessary part of mathematical representation of the physical world.

While the mapping account is supposed to support the common view that mathematical models could represent physical systems even though the physical is fundamentally nonmathematical, it actually undermines it. If the notion of representation is to be adequate, the notion of identity between a mathematical structure and the physical structure it represents has to be sufficiently precise. But it is difficult to see how the notion of identity between mathematical and physical structures could be sufficiently precise for the purposes of modern physics if physical structures did not have a mathematical structure. Thus, it seems that any adequate account of mathematical representation of the physical that is based on the mapping account (or some similar cousin of it) will have to presuppose that the represented physical things have mathematical structures.

\section{On the notion of the physical}

The above argument for the mathematical constitution of the physical is focused on accounts of representation which are based on the mapping account of mathematical representations. There have been various objections to the mapping account (see, for example, Frigg and Nuygen (2018) for criticism of structuralist accounts of representation and references therein). I believe that the main idea of the above argument can be extended to any account of mathematical representation of the physical, as any such account would have to include some aspects of the mapping account as a necessary component. Indeed, it is difficult to see how mathematics could have the potency it has in modern natural science without the mapping account. In any case, there is another

weighty reason for rejecting the idea that the physical is non-mathematical. It is related to 
the question of the nature of the physical, and it is applicable to all accounts of scientific representation, independently of whether they embody the mapping account.

A key motivation for thinking about the physical as non-mathematical is an equivocation between two notions of the physical:

(i) the physical as determined by the theoretical and experimental frameworks of modern physics and, more generally, modern natural science; and

(ii) the physical as some kind of stuff out there, the nature of which is not defined by these frameworks.

The second notion of the physical is too vague and rudimentary to play any significant role in most contemporary scientific applications. In particular, theories and models in physics actually refer to the first notion of the physical, where the physical is constituted by the mathematical in the sense that fundamental features of the physical are in effect mathematical. Indeed, physical objects, properties, relations and laws in modern physics are by their very essence characterized in mathematical terms. Yet, although the gap between the above notions of the physical seems unbridgeable, it is common to assume without a justification that our theories and models, which embody the first notion of the physical, are about the second notion of the physical.

The considerations above suggest that in modern natural science:

(a) The common view - that the physical is ontologically separated from the mathematical - fails to explain how mathematical models/theories could represent the physical.

(b) The mapping account is an essential component of mathematical representations of the physical.

(c) The mapping account requires that physical facts have mathematical structure.

(d) The notion of the physical as fundamentally non-mathematical is too vague and rudimentary to play a significant role in modern natural science.

(e) The mathematical constitution of the physical implies that the physical has a a mathematical structure. Accordingly, granting this constitution, it is possible to make sense of the idea that the mapping account is an essential component of mathematical representations of physical facts.

\section{On the scope of the mathematical constitution of the physical}

So far, I have not considered whether the interpretation of scientific theories/models along realist or instrumentalist lines has relevance for the question of mathematical constitution of the physical. The reason for overlooking this issue earlier on is that the 
philosophical interpretative framework of theories/models in current natural science pertains to the scope rather than the existence of mathematical constitution of the physical. Under both realist and instrumentalist interpretations, the physical phenomena that current theories/models account for are mathematically constituted. The raw data that scientists collect often lack the systematicity, stability, and unity required for constructing these phenomena. The phenomena that our theories/models have to answer for are constructed from raw data in terms of various postulates and statistical inferences which are mathematically constituted. The scope of the mathematical constitution of physical facts beyond the phenomena depends on the interpretative framework. If all the current theories/models in natural science were interpreted as purely instrumental, so that they are not supposed to represent anything beyond the phenomena, the mathematical constitution of the physical would only concern the phenomena; for under such interpretative framework, natural science is not supposed to represent anything beyond physical phenomena. While there are reasons to consider parts of modern natural science as purely instrumental, it is doubtful that there is any adequate understanding of all of it as purely instrumental. Accordingly, it is plausible to conceive the mathematical constitution of the physical in modern natural science as pertaining also to various aspects of the physical reality beyond the phenomena. In any case, in the account of mathematical explanation of physical facts that I now turn to propose the extent of the mathematical constitution of the physical has implications for the scope rather than nature of mathematical explanations of physical facts.

\section{A sketch of a new account of mathematical explanation of physical facts}

In thinking about mathematical explanations of physical facts, it is important to distinguish between:

(I) explanations in which mathematical frameworks, structures, or facts explain physical facts; and

(II) explanations of physical facts that merely appeal to mathematical frameworks, structures, or facts in order to represent physical facts.

The account suggested here aims at the first type of explanations. In these explanations, mathematics plays an essential role, which surpasses its representational role. While this requirement may seem trivial, as we shall see in the next section current accounts of mathematical explanation of physical facts struggle to meet it.

In the proposed account, mathematical explanations of physical facts are of two related kinds. One kind of explanation is a subspecies of physical explanations of physical facts. In physical explanations of physical facts, physical facts are explained by physical facts. In the proposed account, mathematical explanation of physical facts are physical explanations of physical facts that focus on the mathematical constitution of some of the physical facts in the explanans. That is, it is a physical explanation of physical facts that highlights the mathematical constitution of some of the physical facts in the explanans, and by highlighting this constitution it deepens and increases the scope of the 
understanding of the explanandum. The second kind of mathematical explanation of physical facts simply highlights the mathematical constitution of physical facts and, similarly to the first kind of explanation, by highlighting this constitution it deepens and increases the scope of the understanding of these facts.

By physical explanation of physical facts, I shall mean explanations of: how physical facts (could) come about; how physical facts (could) influence or make a difference to other physical facts; how physical patterns or regularities (could) come about; how physical facts, patterns or regularities follow from, or are related to other physical facts, patterns, or regularities; how physical principles/laws and physical facts entail other physical facts; how physical principles/laws entail or are related to other physical principles/laws; etc.

The proposed account is sufficiently open-ended to be based on existing accounts of explanation, such as the D-N, causal, and unification accounts of explanation. However, it cannot be identified with any of these accounts since it requires that the mathematical constitution of some physical facts in the explanans be highlighted. Given the account's openness, it will be easier to demonstrate how it works by analyzing the way it circumvents the difficulties that current accounts of mathematical explanations of physical facts encounter. In the next section, I will consider four such accounts.

Finally, recall that, in this study, the term 'physical facts' is used broadly to include natural facts. The proposed account is supposed to cover mathematical explanations of natural facts. I believe that this account could also be extended to social facts. For, arguably, in current social science there is also a mathematical constitution of various social facts, economic facts being a prime example. But, for lack of space, I leave the consideration of this issue for another opportunity.

\section{On mathematical explanations of physical facts}

Recently, the literature on the mathematical explanation of physical facts has grown steadily and various accounts have been proposed. Here I can only consider some of them, and the choice reflects the way the current study has developed so far. Analyzing putative examples of mathematical explanations of physical facts, I will argue that the structure of the explanation in these accounts is unclear and that they are susceptible to the objection that the mathematical frameworks, structures or facts that they appeal to play a representational rather than explanatory role. I will then show how these accounts could be revised along the lines of the proposed account to circumvent these challenges.

\subsection{On a D-N mathematical explanation of the life cycle of 'periodical' cicadas}

Alan Baker $(2005,2009,2017)$ proposes a D-N-like account of mathematical explanation of physical facts. In presenting the account, he focuses on a natural phenomenon that is drawn from evolutionary biology: the life-cycle of the so-called 'periodical' cicada. Certain species of the North-American cicada share the same kind of unusual life-cycle, 
where the nymph remains in the soil for a lengthy period, and then the adult cicada emerges after either 13 or 17 years, depending on the geographical area. Strikingly, the emergence is synchronized among all the members of a cicada species in any given area. The adults emerge within the same few days, they mate, die a few weeks later and then the cycle repeats itself" (Baker 2005, p. 229). Baker's explanation concentrates on the prime-numbered-year cicada life-cycle and it proceeds as follows (Baker 2005, pp. 230233, 2009, p. 614, 2017, p. 195).

\section{Explanation of the periodical cicada life-cycle}

(1) Having a life-cycle period that minimizes intersection with other (nearby/lower) periods is evolutionarily advantageous. [biological law]

(2) Prime periods minimize intersection (compared to non-prime periods). [number theoretic theorem]

(3) Hence organisms with periodic life cycles are likely to evolve periods that are prime. ['mixed' biological/mathematical law] (from (1) and (2))

(4) Cicadas in ecosystem-type E are limited by biological constraints to periods from 14 to 18 years. [ecological constraint]

(5) Hence cicadas in ecosystem-type E are likely to evolve 17-year periods. (from (3) and (4))

Before turning to discuss this explanation, a few remarks are in place. First, the explanation is actually incomplete. It is based on the implicit assumption that if something is evolutionary advantageous, it is likely to occur. This assumption is required for (1) and (2) to imply (3). Second, in appealing to the D-N explanation, Baker (2005, p. 235 ) broadens "the category of laws of nature to include mathematical theorems and principles, which share commonly cited features such as universality and necessity." Third, whether the above explanation is in fact a good explanation of the periodical cicada life-cycle will not matter for the analysis of the nature of Baker's proposed D-Nlike account of mathematical explanation of physical facts.

Baker's core thesis is that "the cicada case study is an example of an indispensable, mathematical explanation of a purely natural phenomenon" (Baker 2009, p. 614). There have been various objections to this explanation, such as that the choice of mathematical apparatus here is arbitrary (e.g. because of the choice of time units), that the explanation begs the question against nominalism, and that the mathematical facts in the explanation play a representational rather than explanatory role (Melia 2000, 2002, Leng 2005, Bangu 2008, Baker 2009, Daly and Langford 2009, Saatsi 2011, 2016, 2018, Koo 2015). Since my main interest here is to identify the nature of Baker's proposed explanation, I will focus on the last objection.

Daly and Langford (2009, p. 657) argue that 
[i]t is this property of the cicadas' life-cycle duration - this periodic intersection with the life-cycles of certain predatory kinds of organism - that plays an explanatory role in why their life-cycle has the duration it has. Primes have similar properties, and so successfully index the duration. But then so also do various nonprimes: measuring the life-cycle in seasons produces analogous patterns.

That is, it is the concrete facts - the durations of the life-cycles of the cicada and of the other relevant organisms in its environment, and the forces of evolution - that provide the explanation, and the number of units these durations amount to (relative to a given measuring system) only index the duration measured.

Saatsi $(2011,2016)$ expresses a similar objection, proposing that Baker's argument could proceed with an alternative premise to (2) and (3):

$(2 / 3)$ For periods in the range of $14-18$ years the intersection-minimizing period is 17. [Fact about time]

While Daly, Langford and Saatsi have nominalist sympathies, the objection that the mathematical fact in Baker's explanation plays only a representational role has also been submitted by Brown (2012, p. 11) who advocates Platonism.

Baker (2017, p. 198) replies that the above nominalist perspective lacks a scope generality: it is inapplicable to other situations in which the ecological conditions are different. As he points out, this is not only a hypothetical point, as there are subspecies of the periodical cicada with 13-year life cycles. Indeed, $(2 / 3)$ could be generalized into a schema (Saati 2011, p. 152):

$(2 / 3)^{*}$ There is a unique intersection minimizing period $\mathrm{T}_{\mathrm{x}}$ for periods in the range $\left[\mathrm{T}_{1}, \ldots, \mathrm{T}_{2}\right]$ years.

Yet, Baker (ibid., p. 199) notes that while $(2 / 3) *$ has a more general scope, the explanation that this schema provides is less unified and has less depth than the original one. Further, Baker argues that the nominalist perspective also lacks topic generality (ibid., pp. 200-208). A mathematical explanation of physical facts has a higher topic generality if the mathematical facts that are supposed to do the explanation could apply to explanations of other topics. For example, Baker shows that a revised version of the explanation in (1)-(5), which meets scope generality and topic generality, could share the same core as an explanation of why in brakeless fixed-gear bicycles the most popular gear ratios are 46/17, 48/17, 50/17, and 46/15.

Baker argues that: (a) for explanations to have a high level of scope and topic generality, mathematics is indispensable; (b) the interpretation of the mathematical facts in a mathematical explanation of physical facts as representations of physical facts will limit the topic generality of the explanation; and (c) the optimal version of the mathematical explanation of the cicada life-cycle "has an explanatory core that is topic general, and is not 'about' any designated class of physical facts, such as facts about time, or facts about 
durations" (ibid., p. 201). To establish (c), he proposes a generalized explanation of the cicada life-cycle, which I discuss below.

I agree with Baker's observation that the nominalist perspective restricts the level of scope generality and topic generality of explanations. But I don't think that his response meets the objection that, in his account, mathematics only represents the physical facts that actually do the explanation. In what follows, I will present the challenge for Baker's account, first considering the original explanation of the periodical cicada life-cycle and then the generalized version.

Baker's explanation in (1)-(5) is ambiguous because premise (2) is ambiguous. It could be interpreted as:

(2i) a fact about time, i.e. a physical fact; or

(2ii) a theorem about number theory, i.e. a purely mathematical fact.

For the above D-N-like explanation to be valid, it requires (2i) rather than (2ii); since (2ii) is a pure mathematical fact, (1) and (2ii) per se do not imply (3). Thus, it may be argued that it is the physical fact about time in (2i) that explains why the cicadas' lifecycle is likely to be prime, and the mathematical fact in (2ii) only represents the physical fact in (2i); and the impression that (1) and (2ii) entail (3) is due to an equivocation between (2i) and (2ii). The upshot is that Baker fails to demonstrate that the explanation of the periodical cicada life-cycle above is a mathematical explanation of a physical fact. If we interpret premise (2) as a physical fact about the nature of time, i.e. as (2i), the DN-like explanation in (1)-(5) is a physical explanation of a physical fact, and it is not clear what explanatory role the mathematical fact about prime numbers plays. If, on the other hand, we interpret (2) as a purely mathematical fact, i.e. as (2ii), the explanation becomes either invalid or unclear: if the explanation is supposed to be a D-N-like explanation, where the explanandum follows deductively from the explanans, it is invalid; and if the explanation is not intended as a D-N-like explanation, it is not clear what kind of explanation it is.

It may be tempting to reply that the above objection does not apply to Baker's generalized explanation of the cicada life-cycle, where the explanatory core is not 'about' any designated class of physical facts because of its topic generality. Yet, as we shall see below, the generalized explanation is still subject to the same challenge.

Generalized explanation of the periodical cicada life-cycle

(M1) The lowest common multiple (LCM) of two numbers, $m, n$, is maximal if and only if $m$ and $n$ are coprime. [pure mathematical fact]

(UC1) The gap between successive co-occurrences of the same pair of cycle elements of two unit-cycles is equal to the LCM of their respective lengths.

(UC2) Hence any pair of unit-cycles with periods $m$ and $n$ maximizes the gap 
between successive co-occurrences of the same pair of cycle elements if and only if $m$ and $n$ are coprime. (from M1 and UC1)

(M2) All and only prime numbers are coprime with all smaller numbers. [pure mathematical fact]

(UC3) Hence, given a unit-cycle, $p_{m}$, of length $m$ and a range of unit cycles, $q_{i}$, of lengths shorter than $m, p_{m}$ maximizes the gap between successive intersections with each $q_{i}$ if and only if $m$ is prime. (from UC2, M2)

(1G) For periodical organisms, having a life-cycle period that maximizes the gap between successive co-occurrences with periodical predators is evolutionarily advantageous. [biological law]

(2G) Periodical organisms with periodical predators whose life cycles are restricted to multiples of a common base unit can be modeled as pairs of unit-cycles.

(3G) Hence organisms with periodic life-cycles that are exposed to periodic predators with shorter life-cycles, and whose life cycles are restricted to multiples of a common base unit, are likely to evolve periods that are prime. ['mixed' biological/mathematical law] (from UC3, 1G, 2G)

(4G) North American periodical cicadas fit the application conditions stated in premise $(3 \mathrm{G})$.

(5G) Hence periodical cicadas are likely to evolve periods that are prime. (from $3 \mathrm{G}, 4 \mathrm{G}$ )

(6G) Cicadas in ecosystem-type E are limited by biological constraints to periods from 14 to 18 years. [ecological constraint]

(7G) 17 is the only prime number between 14 and 18. [pure mathematical fact]

(8G) Hence cicadas in ecosystem-type $E$ are likely to evolve 17-year periods. (from $5 \mathrm{G}, 6 \mathrm{G}, 7 \mathrm{G})$

As it is not difficult to see, in the above explanation the explanatory core - (M1), (M2), and (UC1) - (UC3) - is topic general. Yet, similarly to the original explanation, one could argue that for the inference from (UC3), $(1 \mathrm{G})$ and $(2 \mathrm{G})$ to $(3 \mathrm{G})$ to be valid, (UC3) has to be interpreted as a proposition about physical systems - all the physical systems that satisfy the requirement of topic generality under consideration - and that the mathematical fact in (UC3) is only a representation of this universal physical fact. Since (UC3) follows from (UC1) and (UC2) (and (M1) and (M2)), for the explanation to be valid, (UC1) and (UC2) also have to be interpreted as propositions about physical systems - again, all the physical systems that satisfy the requirement of topic generality under consideration. Thus, like in the original explanation, the generalized explanation of the periodical cicada life-cycle is subject to the challenge that it is either a physical explanation of a physical fact, invalid, or unclear. 
The objection above can be divided into two related objections. $(\alpha)$ For the conclusion $(8 \mathrm{G})$ to follow from the premises in the generalized explanation of the cicada life-cycle, (UC1) - (UC3) have to be interpreted as propositions about physical facts. Thus, the explanation is a physical explanation of a physical fact, and it is not clear in what sense it is a mathematical explanation of a physical fact. ( $\beta$ ) Like in the original explanation of the cicada life-cycle, it may be argued that mathematics plays a representational rather than explanatory role: it represents the physical facts that actually explains the cicada lifecycle.

The second objection is particularly compelling against those who assume that the physical can be separated from the mathematical. It presupposes that the facts about physical co-occurrences could be separated from the corresponding mathematical facts. Similarly, in the original explanation of the cicada life-cycle, the objection presupposes that the physical fact that prime time periods minimize intersection with other (nearby/lower) periods can be separated from the corresponding mathematical fact. But these presuppositions are unwarranted for those who take the mathematical to constitute the physical. Granted the mathematical constitution of the physical, the physical fact that prime time periods minimize intersection with other (nearby/lower) periods is constituted by number theory, in general, and its theorems about prime numbers, in particular. Accordingly, the physical fact that prime time periods minimize intersection with other (nearby/lower) periods cannot be separated from the corresponding mathematical fact. Likewise, facts about physical co-occurrences are constituted by number theory, in general, and its theorems about prime numbers, in particular. Thus, such facts cannot be separated from the corresponding mathematical facts.

Granted the mathematical constitution of the physical, Baker's D-N-like account of mathematical explanation of physical facts can be revised as follows to circumvent the objections in $(\alpha)$ and $(\beta)$. A mathematical explanation of physical facts is a physical explanation of physical facts along the D-N model in which the mathematical constitution of some physical facts in the explanans is highlighted. The idea here is that by highlighting the mathematical constitution of certain physical facts in the explanans, the explanation deepens and expands the scope of the understanding of the physical fact in the explanandum. Focusing on the original explanation of the periodical cicada life-cycle, the revised account has two parts. First, it derives deductively the likelihood of the primenumbered-year life-cycle of the periodical cicada from general biological facts, particular facts about the periodical cicada and its eco-system, and facts about physical time. Second, it highlights the mathematical constitution of physical time, in general, and the mathematical constitution of the fact that prime periods of physical time minimize intersection with other time periods, in particular. Indeed, the theorem of number theory in (2ii) is a reflection of this constitution. Time is modelled as a line of real numbers and accordingly time intervals with a length equal to a natural number are subject to the theorems of number theory. In highlighting the mathematical constitution of these physical facts, the explanation deepens our understanding of the curious life-cycle of the periodical cicada in eco-system type $\mathrm{E}$ and expands the potential scope of the explanation to the life-cycle of other subspecies of the periodical cicada. Turning to the generalized explanation of the periodical cicada life-cycle, in addition to showing how the likelihood 
of the prime-numbered-year life-cycle of the periodical cicada follows deductively from general biological facts, particular facts about the periodical cicada and its eco-system, and universal physical facts about successive co-occurrences of the same pair of cycle elements of two-unit cycles, the explanation highlights the mathematical constitution of these latter physical facts. In highlighting these facts, the explanation deepens the understanding of the periodical cicada life-cycle in ecosystem-type E and expands its potential scope to explanations of other subspecies of the periodical cicada - namely, periodical cicada in other types of ecosystems - as well as to explanations of various other natural facts.

As it is not difficult to see, the proposed revision of Baker's account of mathematical explanation of physical facts is not subject to the objections in $(\alpha)$ and $(\beta)$. Premise (2) in the original explanation of the periodical cicada life-cycle and premises (UC1) - (UC3) in the generalized explanation of this life-cycle are interpreted as statements about intervals of time and physical unit-cycles, respectively. Thus, the objection that the explanans in these explanations do not imply their explanandum does not apply. Further, the logical structure of the explanation is clear, and, by construction, mathematics plays an explanatory role.

\subsection{On structural explanation of the uncertainty relations}

Dorato and Felline (2011, p. 161) comment that the ongoing controversy concerning the interpretation of the formalism of quantum mechanics may explain why philosophers have often contrasted the poor explanatory power of quantum theory to its unparalleled predictive capacity. Yet, they claim, "quantum theory provides a kind of mathematical explanation of the physical phenomena it is about", which they refer to as structural explanation. To demonstrate their claim, they present two case studies: one involves the quantum uncertainty relations between position and momentum, and the other focuses on quantum nonlocality.

Following Clifton (1998, p. 7) ${ }^{7}$, Dorato and Felline (2011, p. 163) hold that

we explain some feature $\mathrm{B}$ of the physical world by displaying a mathematical model of part of the world and demonstrating that there is a feature A of the model that corresponds to B, and is not explicit in the definition of the model.

The idea here is that the explanandum $\mathrm{B}$ is made intelligible via its structure similarities with its formal representative, the explanans A. How do these structural similarities render the explanandum intelligible? Dorato and Felline propose that "in order for such a representational relation to also be sufficient for a structural explanation, ... we have to accept the idea that we understand the physical phenomenon in terms of its formal representative, by locating the latter in the appropriate model” (ibid., p. 165).

\footnotetext{
${ }^{7}$ Clifton (1998) appropriates, with minor modifications, a definition of explanation given by Hughes (1993).
} 
Consider, for example, the uncertainty relations between position and momentum. In standard quantum mechanics, systems' position and momentum are related through Fourier transforms, and Dorato and Felline locate the explanans as the mathematical properties of the Fourier transforms that represent the relations between position and momentum. The formal representation of the momentum (position) of a particle is a Fourier transform of the formal representation of its position (momentum), and Dorato and Felline propose that these Fourier transforms are required to make intelligible the relevant, represented physical phenomena of the uncertainty relations between position and momentum. These transformations are supposed to constitute the answer to the question "why do position and momentum not assume simultaneously sharp magnitudes?": "because their formal representatives in the mathematical model have a property that makes this impossible" (ibid., p 166).

Since 'because' here is not supposed to have a causal interpretation, the question arises as to its exact meaning. Dorato and Felline do not address this question explicitly. Their structural account of explanation seems to be based on the following key ideas: (i) A structural explanation makes the explanandum understandable; (ii) we understand a physical fact by making it intelligible; (iii) the assumption that the properties of a physical system exemplify the relevant parts of the mathematical model that represents it allows one to use the properties of the latter to make intelligible the properties of the former; (iv) there is a structure-preserving morphism from the representing mathematical model to the represented physical fact, and this relation ensures that the represented fact can be made intelligible by locating its representative in the mathematical model. Yet, these ideas leave the logical structure of the structural explanation unclear. In particular, the extent to which the structural explanation is different from mere representation is unclear. Indeed, as Dorato and Felline acknowledge, one may raise the objection that the proposed explanation is a mere translation or redescription of the physical explanation to be given (ibid., p. 166). They concede that, in a sense, it is the physical properties that do the explanation. They consider, for example, a balance with eight identical apples, five on one pan and three on the other.

If someone explained the dropping of the pan with five apples (or the rising of the side with three) by simply saying " $5>3$ ", he/she would not have provided a genuine explanation. The side with five apples drops because it is heavier and because of the role that its gravitational mass has vis-à-vis the earth, not because $5>3$ !

In reply, Dorato and Felline claim that "structural explanations are not so easily translatable into non-mathematical terms without loss of explicative power" (ibid., p. 167). The problem with this reply is that it does not really address the above objection. The question under consideration is not whether to replace mathematical explanations with non-mathematical ones. Rather, it is a question about the nature of the mathematical explanation on offer. In particular, it is not clear in what sense the proposed structural explanation is a mathematical explanation of physical facts rather than a physical explanation of physical facts in which mathematics only plays a representational role. For 
example, focusing on the explanation of the uncertainty relations, it is not clear why rendering the uncertainty relations between position and momentum intelligible by pointing to the Fourier transforms between the functions that represent position and momentum is a mathematical explanation of a physical fact rather than a physical explanation of a physical fact in which mathematics only plays a representational role.

Another aspect that is unclear in Dorato and Felline's structural explanation is related to the question whether the proposed pattern of explanation requires an interpretation of the representing mathematical model. Dorato and Felline conceive the structural explanation as providing "a common ground for understanding [the uncertainty relations between position and momentum], independently of the various different ontologies underlying the different interpretations of quantum theory" (ibid., p. 165). The question arises then: How does this conception square with the fact that their structural explanation relies on the idea that for a mathematical model to explain properties of a physical system the physical system has to exemplify the relevant parts of the model? The problem here is that the quantum-mechanical formalism represents different things under different interpretations. Consider, for example, Bohmian mechanics (Goldstein 2017). The functions that represent systems' positions and momentum in the standard interpretation of the quantum-mechanical formalism do not represent their position and momentum in Bohmian mechanics. Under this alternative interpretation, quantum systems always have definite position and momentum, and systems' position and momentum do not exemplify the Fourier transform relations. Thus, the explanation that the quantum-mechanical formal representatives - the Fourier transforms between position and momentum - have a property that makes it impossible for systems to have simultaneously definite position and momentum is not correct in this case. The functions that represent systems' position and momentum in the standard interpretation represent in Bohmian mechanics the range of possible outcomes of measurements of positions and momentum and their probabilities; and the Fourier transform relations between these functions reflect the epistemic limitation on the knowledge of systems' position and momentum. The upshot is that Dorato \& Felline 's structural explanation cannot circumvent the question of the interpretation of the quantum-mechanical formalism.

Granted the mathematical constitution of the physical, Dorato \& Felline's structural account of explanation can be revised along the lines proposed in Section 8 so as to meet the above challenges. The main idea of the revised account is that a reference to the mathematical representatives of physical facts is explanatory if it highlights the mathematical constitution of these facts and thus deepen and potentially extend the scope of our understanding of them. For example, the statement that, in standard quantum mechanics, position and momentum cannot assume simultaneously definite values "because their formal representatives in the mathematical model have a property that makes this impossible" is explanatory because it highlights the mathematical constitution of the relations between position and momentum and thus deepen our understanding of these relations. In Bohmian mechanics, a reference to these formal representatives also highlights the mathematical constitution of physical facts, but the physical facts are not the same as in standard quantum mechanics. In Bohmian mechanics, the highlighting is 
of the mathematical constitution of the limitation on simultaneous measurements, and accordingly knowledge, of a system's position and its momentum at any given time. $>>>$

\subsection{On abstract mathematical explanation of the impossibility of a minimal tour across the bridges of Königsberg}

Pincock (2007, 2011a,b, 2012, 2015) proposes an account of mathematical explanation which he calls abstract explanation. Like most causal accounts of explanation, abstract explanation is based on the idea of an objective dependence relation between the explanandum and the explanans, but the notion of dependence in play is different from the causal notion of dependence (Pincock 2015, p. 877). In the case of abstract explanation, the dependence is on an abstract entity which is a more abstract than the state of affairs being explained (ibid., p. 879). Pincock has applied this account to various examples, one of which is the explanation of the impossibility of a 'minimal' tour across the bridges of Königsberg.

The citizens of 18th C Königsberg wished to make a minimal tour across the city's bridges (see Fig. 1), crossing each bridge exactly once and returning to the starting point. But they failed. Euler saw that the network of bridges and islands yield a certain abstract structure. Represented in graph theory, this structure has edges which correspond to bridges and vertices which correspond to islands or banks (see Fig. 2). Let us call a graph 'Eulerian' just in case it has a continuous path from a vertex that crosses each edge exactly once and return to that vertex (for an example of such a graph, see Fig. 3). Then, Euler's (1736/1956) conclusions about minimal tours across bridge systems can be reformulated as the following theorem in graph theory. ${ }^{8}$

Euler's theorem: A graph is Eulerian if and only if it contains no odd vertices; where a vertex is called 'odd' just in case it has an odd number of edges leading to it.

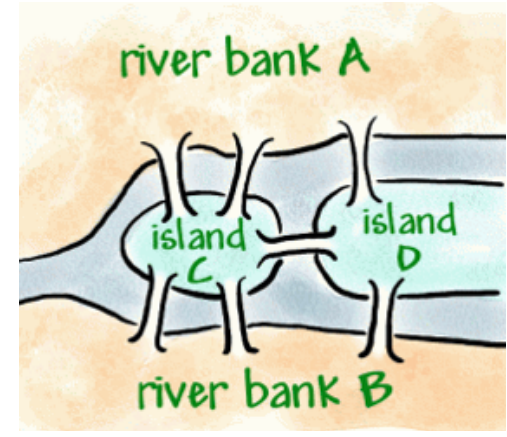

Fig. 1: The bridges of Königsberg

\footnotetext{
${ }^{8}$ Since graph theory did not exist at the time, this is obviously an anachronistic account of Euler's reasoning. That is not a problem for the current discussion, as the aim is not to reconstruct Euler's own analysis but rather to consider Pincock's account of mathematical explanation of physical facts. For Euler's reasoning, see Euler (1736/1956), and for a reconstruction of it, see, for example, Hopkins and Wilson (2004).
} 


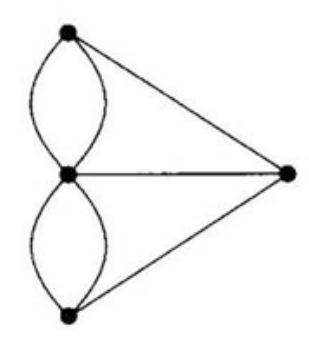

Fig. 2: The graph of the bridges of Königsberg: Edges correspond to bridges and vertices correspond to islands or banks

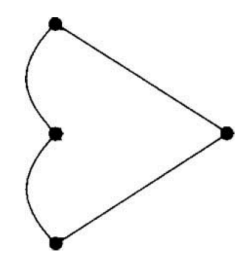

Fig. 3: An example of Eulerian graph

Pincock's proposed abstract explanation for the impossibility of a minimal tour across the bridges of Königsberg is that such a tour is impossible because the corresponding graph is not Eulerian. Put another way, the impossibility of a minimal tour across the bridges of Königsberg is abstractly dependent on the corresponding graph not being Eulerian.

Pincock's motivation for an abstract explanation of the impossibility of a minimal walk across the Königsberg bridge system is that it seems superior because it gets to the root cause of why such a walk is impossible by focusing on the abstract structure of this system. More generally, abstracting away from various physical facts, such as the bridge materials and dimensions, size of the islands and river banks, etc., scientists can often give better explanations of features of physical systems (Pincock 2007, p. 260).

Since abstract dependence is supposed to be objective but not causal, the question arises as to what kind of dependence it is. Pincock considers a few candidates. First, he (2015) discusses Woodward's (2003, p. 221) proposal that

the common element in many forms of explanation, both causal and noncausal, is that they must answer what-if-things-had-been-different questions. ... When a theory or derivation answers a what-if-things-had-been-different question but we cannot interpret this as an answer to a question about what would happen under an intervention, we may have a noncausal explanation of some sort.

In the case of Pincock's abstract account of explanation, the question that is answered concerns the "the systematic relationship between the more abstract objects and their properties [the explanans], and the more concrete objects and their properties [the explanandum]." Pincock comments that Woodward's proposal could be applicable to 
abstract explanations "if the sort of explanatory question here could be suitably clarified" (ibid., p. 869).

Next, Pincock (ibid., p. 869-871) discusses Strevens' (2008) Kairetic account of explanation. He notes that the Kairetic account "allows important explanatory contributions from more abstract entities like mathematical objects." He considers whether this account - which, like Woodward's account, is based on the idea of difference making, and moreover aims to reduce all explanations to causal explanations could be a candidate for explicating the notion of abstract dependence. Pincock rejects this route on the ground that abstract dependence violates Strevens' 'cohesion' condition; where a causal model is cohesive "if its realizers constitute a contiguous set in causal similarity space" (Strevens 2008, p. 101).

Pincock (2011) also considers a notion of difference making which is explicated in terms of comparisons across relevant range of possibilities. Applying this notion to the explanation of why a minimal tour across the bridges of Königsberg is impossible, his reasoning is basically as follows. Let's $V$ be a binary variable that denote whether all vertices of a graph are even, $E$ be a binary variable that denote whether the graph is Eulerian, and $M$ be a binary variable that denote whether there is a minimal tour across the bridges of Königsberg. The graph of the bridges of Königsberg fails to be Eulerian, but many similar graphs have all even valence vertices and each of these graphs is Eulerian. Thus, $V$ makes a difference for $E$. Further, the bridges of Königsberg fail to have a corresponding graph that is Eulerian and they fail to have a minimal tour, but many similar bridge systems have a corresponding graph that is Eulerian and each of these other bridge systems has a minimal tour. Thus, $E$ makes a difference for $M$.

In the above proposal, the exact nature of abstract dependence is still unclear. In particular, Pincock does not explicate the type of similarity he has in mind, so it is difficult to evaluate the nature of modality involved in his proposed notion of difference making. Relatedly, it may be argued that Pincock's reasoning does not establish that $E$ $(V)$ makes a difference for $M$ but rather that there is a correspondence between $E(V)$ and $M$, and that such a correspondence per se does not qualify as a difference making. It is also unclear why one needs to appeal to the notion of difference making in order to establish the modal relation between $V$ and $E$ given that one could simply provide a mathematical proof of Euler's theorem.

Finally, Pincock (2015) considers Koslicki's (2012) notion of ontological dependence as a possible route to explicating the nature of abstract dependence. One variety of such a dependence is the following.

Constituent Dependence: An entity, $\Phi$, is constituent dependent on an entity (or entities), $\Psi$, just in case $\Psi$ is an essential constituent (or are essential constituents) of $\Phi$. (Koslicki, ibid., p. 205)

An example of such ontological dependence is lightning: "for lightning to occur is just for energy to be discharged by some electrons in a certain way, and when lightning 
occurs, these electrons are constituents of the lightning" (Pincock 2015, p. 878). Pincock (ibid., p. 879) remarks that while "it might be tempting to try to reduce abstract dependence to constituent dependence" and "[it] is hard to argue that this cannot be done." But

there is one prima facie barrier that seems difficult to overcome. ... [a] distinguishing feature of abstract explanation and abstract dependence is that we appeal to a more abstract entity that has a more concrete entity as an instance. ... By contrast, in the constituent cases, the entities that we appeal to in the explanation are constituents of the fact to be explained.

As the brief review of Pincock's attempts to explicate abstract dependence demonstrates, the nature of this dependence remains unclear. Further, the abstract account of explanation is open to the objection that mathematics just plays a representational role. The idea here is that the graph that corresponds to the bridges of Königsberg only represents the relevant physical structure of this bridge system which explains the impossibility of a minimal tour (Janssen and Saatsi, forthcoming). ${ }^{9}$ This objection has more traction in view of the difficulties in explicating abstract dependence, and it is even more pressing for those who deny the mathematical constitution of the physical; for it is difficult to see how the impossibility of a minimal tour - a physical fact - could depend on a mathematical object - a graph - if the physical is fundamentally non-mathematical.

While the prospects of explicating abstract dependence in terms of Constituent Dependence seem dim, there are other notions of ontological dependence that escape this fate. In particular, the notions of mathematical constitution of physical facts we reviewed in Section 4 are not subject to Pincock's concern about Constituent Dependence. When abstract dependence involves a dependence of physical facts on mathematical structures or facts, it could be explicated in terms of these notions of mathematical constitution. Consider, again, the bridges of Königsberg. The impossibility of a minimal tour across the Königsberg bridge system is due to its physical structure. This physical structure has a mathematical constitution. Some aspects of this constitution, which concern the topology of the Königsberg bridge system, are highlighted by graph theory, in general, and Euler's theorem, in particular. Thus, abstract explanation of the impossibility of a minimal tour across the Königsberg bridge system could be conceived as highlighting the aspects of the mathematical constitution of the physical structure of this bridge system that render such a tour impossible. Envisaged in this way, the explanation highlights the ontological dependence of the physical structure of the Königsberg bridge system on a certain mathematical structure, which is expressed in terms of graph theory. Highlighting the relevant aspects of the mathematical constitution of this physical structure deepens our understanding of the impossibility of a minimal tour across it. It also increases the scope

\footnotetext{
${ }^{9}$ Vineberg (2018) suggests that the objection above does not apply to the structuralist understanding of mathematics. It is not clear, however, how an appeal to this conception of mathematics could help here. One may accept the structuralist rejection of mathematical objects yet argue that mathematical structures only represent the physical structures which actually do the explanation.
} 
of our understanding by relating this impossibility to various other cases of possible and impossible minimal tours.

\subsection{On explanations by constraints that are more necessary than laws of nature}

In Because without cause: Non-causal explanations in science and mathematics, Lange (2016) considers examples of "distinctively mathematical explanations" that are of the kind we discussed above. For him, distinctively mathematical explanations are subspecies of a more general type of non-causal explanation: "explanations by constraint".

Explanations by constraint work not by describing the world's causal relations, but rather by describing how the explanandum arises from certain facts ("constraints") possessing some variety of necessity stronger than ordinary laws of nature possess. The mathematical facts figuring in distinctively mathematical explanations possess one such stronger variety of necessity: mathematical necessity. (Ibid., p. 10)

Lange suggests that a distinctive mathematical explanation appeals "only to facts (including but not always limited to mathematical facts) that are modally stronger than ordinary laws of nature, together with contingent conditions that are contextually understood to be constitutive of the arrangement or task at issue in the why question" (ibid., pp. 9-10). That is, in such explanations the explanans consist of mathematically necessary facts and possibly other facts that possess some variety of necessity stronger than laws of nature possess, as well as contingent conditions which are presupposed by the why question under consideration. The explanation shows that "the fact to be explained could not have been otherwise - indeed, was inevitable to a stronger degree than could result from actions of causal powers" (ibid., pp. 6-7). Under the presupposed contingent conditions, the explanadum arises from the mathematical facts and possibly other necessary facts (the "constraints"), and it is necessary in a stronger sense than the necessity that ordinary laws of nature mandate. Thus, the explanation works

not by describing the world's actual causal structure, but rather by showing how the explanandum arises from the framework that any possible physical system (whether or not it figures in causal relations) must inhabit, where the "possible" systems extend well beyond those that are logically consistent with all of the actual natural laws. (Ibid., pp. 30-31)

For example, the fact that a mother failed repeatedly to distribute evenly 23 strawberries among her 3 children without cutting any strawberry is explained by the mathematical fact that 23 cannot be divided evenly by 3 (ibid., p. 6). The explanation shows that mother's success is impossible in a stronger sense than causal considerations underwrite (ibid., p. 30). The explanans in this case consist of the mathematical fact that 23 cannot be divided evenly by 3 and the contingent facts presupposed by the relevant whyquestion: namely, that there are 23 strawberries and 3 children, and the distribution is of uncut strawberries. And the explanation shows that, under such contingent conditions, even distribution among the 3 children is impossible, where the impossibility is stronger than natural impossibility. 
Saatsi (2018) poses two challenges for Lange's account of explanation. One challenge is that "information about the strong degree of necessity involved [in explanation by constraint] risks being too cheap: the exalted modal aspect of the explanandum can be communicated without doing much explaining, and it can be grasped without having much understanding" (ibid., p. 5). Another challenge is to pin down the difference due to which non-causal explanations-by-constraint work so differently from causal explanations. Saatsi proposes, as an alternative to Lange's approach, that causal and noncausal explanations alike "can explain by virtue of providing what-if-things-had-beendifferent information that captures a dependence relation between the explanandum and the explanans." He believes that this counterfactual-dependence perspective suggests that, in various distinctively mathematical explanations, at the very least "we should not hang the analysis of explanatoriness entirely on the hook of modal 'constraint'." For example, "if we squeeze out, as it were, all the modal information regarding how Mother's predicament would differ as a function of the number of strawberries/kids, it looks that we are left with a very shallow explanation at best, even if we fully retain the information concerning the exalted modal status of the explanandum" (ibid., p. 6).

Lange (2016, 2018, p. 32) resists Saatsi's proposal for understanding distinctively mathematical explanations in terms of counterfactual dependence. He maintains that some explanations by constraint, and more particularly some distinctively mathematical explanations, are associated with no pattern of counterfactual dependence. Further, he (2018, p. 33) also argues that "[s]ometimes the explanans and explanandum of explanations by constraint do figure in patterns of counterfactual dependence, but these patterns fail to track explanatory relations". Yet, while Saatsi's view that causal and noncausal explanations could be accounted in terms of counterfactual dependence is controversial, his objections highlight the fact that the exact nature of Lange's distinctively mathematical explanations is unclear. Consider, for example, Saatsi's question:

Why is it that given that mass is additive, if $\mathrm{A}$ has the mass of $1 \mathrm{~kg}$, and $\mathrm{B}$ has the mass of $1 \mathrm{~kg}$, then the union $\mathrm{A}+\mathrm{B}$ has the mass of $2 \mathrm{~kg}$ ?

Lange (2018, p. 35) admits that "perhaps Saatsi is correct that the answer 'Because $1+1=2$ ' is 'utterly shallow'.' But he suggests that

that impression may arise from everyone's knowing that $1+1=2$ and that this fact suffices to necessitate the explanandum, so it is difficult to see what information someone asking Saatsi's question might want. Furthermore, "Because 1+1=2", may not explain $A+B$ 's mass at all, if $A$ and $B$ can chemically interact when "united". (Ibid.)

Indeed, the mathematical fact $1+1=2$ and its intuitive relation to the explanandum are very familiar. But this familiarity conceals the fact that the exact structure of Lange's account of distinctively mathematical explanation is unclear. That is, it is not clear from Lange's account how the "because" of explanation is supposed to work: why a mathematical fact per se could explain facts about the nature of physical objects? One 
may argue here that the fact that $1+1=2$ seems to necessitate the explanandum is due to equivocation between: (i) the mathematical fact that $1+1=2$; and (ii) the universal physical fact that, for any two possible physical objects of $1 \mathrm{~kg}, \mathrm{~A}$ and $\mathrm{B}$, which are 'united' without being changed (e.g. without having a chemical interaction), the total mass is $2 \mathrm{~kg}$. That is, one may argue that it is the physical fact in (ii), rather the mathematical fact in (i), that actually does the explanation, and that the mathematical fact in (i) just represents the physical fact in (ii). Put another way, one may argue that the intuitive appeal of Lange's distinctively mathematical explanation is due to the above equivocation, taking the explanation to be a physical explanation of a physical fact - it is an explanation of a particular physical fact in terms of a corresponding universal physical fact - in which the mathematical fact $1+1=2$ only plays a representational role.

The above objection is particularly compelling for those who maintain that the physical is ontologically separated from the mathematical. It can be circumvented by acknowledging the mathematical constitution of the physical. Distinctively mathematical explanations could then be understood as physical explanations of physical facts in which the mathematical constitution of some physical facts is highlighted by stating the constraints that this constitution mandates. In such explanations, there is no need to appeal to counterfactual dependence. For example, in explaining "why the union A+B has the mass of $2 \mathrm{~kg}$ ?" by pointing out that " $1+1=2$ ", one appeals to the universal physical fact that for any two possible physical objects of $1 \mathrm{~kg}, \mathrm{~A}$ and $\mathrm{B}$, which are 'united' without being changed, the total mass of $\mathrm{A}+\mathrm{B}$ is $2 \mathrm{~kg}$. Yet, one observes that this universal physical fact is constituted by number theory, in general, and a mathematical fact that follows from it, $1+1=2$, in particular. There is no need to appeal to counterfactual dependence. Indeed, it is not clear how counterfactual dependence could be of any help in this kind of explanation.

\section{Is the effectiveness of mathematics in physics unreasonable?}

It seems to be a dogma of contemporary mainstream philosophy of science that, fundamentally, physical facts are not mathematical, and that mathematics only provides a language for representing the physical realm, even if this language is indispensable. Thus, the idea of mathematical explanations of physical facts may naturally appear puzzling or even paradoxical. I argued above that the view that the physical is ontologically separated from the mathematical fails to make sense of the common view of how mathematical models and theories represent physical phenomena and reality in modern natural science. I suggested that this conundrum could be avoided if we accept the idea that the physical is constituted by the mathematical. I then reviewed two traditional ways of conceiving such a constitution: the Pythagorean and the neo-Kantian. Granted the mathematical constitution of the physical, I proposed a new account of mathematical explanation of physical facts. In this account, there are two related kinds of explanations. One kind of mathematical explanation of physical facts consists in: (E) explaining physical facts by physical facts along the lines of the D-N, causal, unification, or any other acceptable account of explanation; and $(\mathrm{H})$ highlighting the mathematical constitution of some of the physical facts that do the explanation in (E) and thus deepening and expanding the scope of the understanding of the explained physical facts. The second kind of mathematical 
explanation of physical facts consists in (H). I also considered four other accounts of mathematical explanation of physical facts. I argued that, unlike the proposed account, they are open to the challenge that the nature of their explanation is unclear and the objection that mathematics plays only a representational role, representing the physical facts that actually do the explanation. I then suggested that these accounts could be revised along the lines of the proposed account so as to circumvent both challenges.

The proposed account is neutral with respect to the controversy in the philosophy of mathematics about the ontological status of 'mathematical objects' (numbers, sets, relations, functions, etc.). Further, it applies to both realist and instrumentalist interpretations of theories/models. The interpretation of the ontological status of theoretical terms only determines the scope of the mathematical constitution of the physical beyond the phenomena.

In conclusion, I turn briefly to comment on how the idea of the mathematical constitution of the physical reflects on the question whether the effectiveness of the use of mathematics in physics is unreasonable. It is reasonable to assume that one's conception of mathematics and its relation to physics is important for adjudicating this question. Let us consider then Wigner's conception. Wigner (1960) holds that while

the concepts of elementary mathematics and particularly elementary geometry were formulated to describe entities which are directly suggested by the actual world, the same does not seem to be true of the more advanced concepts, in particular the concepts which play such an important role in physics. ... Most more advanced mathematical concepts, such as complex numbers, algebras, linear operators, Borel sets - and this list could be continued almost indefinitely - were so devised that they are apt subjects on which the mathematician can demonstrate his ingenuity and sense of formal beauty.

He conceives mathematics as

the science of skillful operations with concepts and rules invented just for this purpose. The principal emphasis is on the invention of concepts. ... The depth of thought which goes into the formulation of the mathematical concepts is later justified by the skill with which these concepts are used. The great mathematician fully, almost ruthlessly, exploits the domain of permissible reasoning and skirts the impermissible. That his recklessness does not lead him into a morass of contradictions is a miracle in itself ... The principal point which will have to be recalled later is that the mathematician could formulate only a handful of interesting theorems without defining concepts beyond those contained in the axioms and that the concepts outside those contained in the axioms are defined with a view of permitting ingenious logical operations which appeal to our aesthetic sense both as operations and also in their results of great generality and simplicity.

Based on this conception and consideration of the application of various "advanced mathematical concepts" in physics, Wigner concludes that the appropriateness of the 
language of mathematics for the formulation of the laws of physics is a miracle, and he hopes that this miracle will continue in future research.

On Wigner's conception of mathematics, the relation between the physical and mathematical is not intrinsic. Advanced mathematics largely develops on its own and is then picked up by physicists to yield great success. It may thus be natural to see the success of the application of the language of mathematics in physics as surprising, mysterious, and even unreasonable. However, Wigner's conception is controversial (see, for example, Lützen 2011, Ferreirós 2017, Islami 2017, and references therein). Some have argued that while Wigner's view of mathematics seems to have been inspired by the formalist philosophy of mathematics, this philosophy has lost its credibility by the second half of the $20^{\text {th }}$ century (Lützen 2011, Ferreirós 2017). Further, historically, the development of mathematics has been entangled with that of the natural sciences, in general, and physics, in particular, and Wigner's conception of mathematics fails to reflect this fact. Consider, for example, complex numbers. Wigner singles them out as one of the prime examples of most advanced mathematical concepts that mathematicians invented for the sole purpose of demonstrating their ingenuity and sense of formal beauty without any regard to possible applications. Indeed, it seems that complex numbers were introduced by Scipione del Ferro, Niccola Fontana (also known as Tartaglia, the stammerer), Gerolamo Cardano, Ludovico Ferrari and Rafael Bombelli in the $16^{\text {th }}$ century with no intent in mind to apply them. Yet, the context of the introduction was the attempt to solve the general forms of quadratic and cubic equations, which by that time had a long history of applications (Katz 2009). Thus, taking into account this broader context, the claim that the introduction of complex numbers was solely for the purpose of demonstrating ingenuity and sense of formal beauty is misleading.

That is not to argue that all the developments in mathematics were connected to physics, neither to deny that in many cases future applications of mathematical concepts could not be anticipated at the time of their introduction. Yet, attention to the historical entanglement between the developments of mathematics and physics casts doubt on the validity and scope of Wigner's argument for the unreasonable success of the application of mathematics in physics.

In any case, while Wigner's view of mathematics may provide some support to the view that the success of the application of mathematics in physics is unreasonable, things are very different if we conceive the mathematical as constitutive of the physical. In the context of such a conception, the relationship between mathematics and physics is intrinsic. The physical is characterized in mathematical terms. The physicist's crude experience is formulated in precise mathematical terms, often in statistical models of the phenomena, and accordingly the gap between the phenomena and the theoretical models that account for them diminishes. There is no essential gap between the concepts of elementary mathematics and geometry and more advanced mathematical concepts. The division between applied and theoretical mathematics is neither a priori nor fundamental. And the appropriateness of the use of the language of mathematics for future physics is not in doubt. Thus, it is reasonable to expect the language of mathematics to be appropriate for representing physical reality. 
Of course, such a conception of the role of mathematics in physics does not obliterate the sense of wonder that one has with respect to the fact that "in spite of the baffling complexity of the world, certain regularities in the events could be discovered" (Wigner, ibid.). Yet, the reasons to conceive this wonder in miraculous terms are not as compelling as for those who deny the mathematical constitution of physical facts.

\begin{abstract}
Acknowledgments. I owe a great debt to Itamar Pitowsky. Itamar's graduate course in the philosophy of probability stimulated my interest in the philosophical foundations of probability and quantum mechanics. Itamar supervised my course essay and MA thesis on the application of de Finetti's theory of probability to the interpretation of quantum probabilities. During my work on this research project, I learned from Itamar a great deal about the curious nature of probabilities in quantum mechanics. Itamar was very generous with his time and the discussions with him were always enlightening. Our conversations continued for many years to come, and likewise they were always helpful in clarifying and developing my thoughts and ideas. Itamar's untimely death has been a great loss. Whenever I have an idea I would like to test, I think of him and wish we could talk about it. I sorely miss the meetings with him and I wish I could discuss with him the questions and ideas considered above.

I am very grateful for the volume editors, Meir Hemmo and Orly Shenker, for inviting me to contribute, and for Meir for drawing my attention to Itamar's commentary on the relationship between mathematics and physics. The main ideas of the proposed account of mathematical explanations of physical facts were first presented in the workshop on Mathematical and Geometrical Explanations at the Universitat Autònoma de Barcelona (March 2012), and I thank Laura Felline for inviting me to participate in the workshop. Earlier versions of this paper were also presented in the $39^{\text {th }}, 42^{\text {nd }}$ and $46^{\text {th }}$ Dubrovnik Philosophy of Science conferences, IHPST, Paris, IHPST, University of Toronto, Philosophy, Università degli Studi Roma Tre, Philosophy, Università degli Studi di Firenze, CSHPS, Victoria, CPNSS, LSE, Philosophy, Leibniz Universität Hannover, ISHPS, Jerusalem, Munich Center for Mathematical Philosophy, LMU, Faculty of Sciences, Universidade de Lisboa. I would like to thank the audiences in these venues for their helpful comments. For discussions and comments on earlier drafts of the paper, I am very grateful to Jim Brown, Laura Felline, Aaron Kenna, Flavia Padovani, Noah Stemeroff, and anonymous referee. The research for this paper was supported by SSHRC Insight and SIG grants as well as Victoria College travel grants.
\end{abstract}

\title{
References
}

Aristotle. Metaphysics. A revised text with introduction and commentary by W.D. Ross. Two vols. Oxford: Clarendon Press, 1924.

Bachelard, G. 1965. L'activité rationaliste de la physique contemporaine. Paris: Presses Universitaires de France.

Baker, A. 2005. Are there genuine mathematical explanations of physical phenomena? Mind 114(454), 223-238. 
Baker, A. 2009. Mathematical explanations in science. British Journal for the Philosophy of Science 60(3):611-633

Baker, A. 2017. Mathematics and explanatory generality. Philosophia Mathematica 25(2), 194209.

Bangu, S. 2008. Inference to the best explanation and mathematical explanation. Synthese 160(1), 13-20.

Baron, S., Colyvan, M. and Ripley, D. 2017. How mathematics can make a difference. Philosophers' Imprint 17(3), 1-19.

Batterman, R. 2002. Asymptotics and the role of minimal models. British Journal for the Philosophy of Science 53(1), 21-38.

Batterman, R. 2010. On the explanatory role of mathematics in empirical science. British Journal for the Philosophy of Science, 61(1), 1-25.

Batterman, R. 2018. Autonomy of theories: An explanatory problem. Noûs 52(4), 858-873.

Batterman, R. and Rice, C. 2014, Minimal model explanations. Philosophy of Science 81(3), 349-376.

Bell, J. S. 1987. Speakable and unspeakable in quantum mechanics. Cambridge: Cambridge University Press.

Bokulich, A. 2008a. Can classical structures explain quantum phenomena? British Journal for the Philosophy of Science 59(2), 217-35.

Bokulich, A. 2008b. Reexamining the quantum-classical relation: Beyond reductionism and pluralism. Cambridge: Cambridge University Press.

Bokulich, A. 2011. How scientific models can explain? Synthese 180(1), 33-45.

Bolzano, B. 1930. Functionenlehre. In K. Rychlik (ed.), Royal Bohemian Academy of Sciences, Prague.

Brown, J. R. 2012. Plantonism, naturalism, and mathematical knowledge. London: Routledge.

Bueno, O. and Colyvan, M. 2011. An inferential conception of the application of mathematics. Nous 45, 345-374.

Bueno, O. and French, S. 2018. Applying mathematics: Immersion, inference, interpretation. Oxford: Oxford University Press.

Cassirer, E. 1907. Kant und die moderne mathematik. Kant-Studien 12, 1- 49.

Cassirer, E. 1910. Substanzbegriff und funktionsbegriff. Untersuchungen über die Grundfragen der Erkenntniskritik. Berlin: Bruno Cassirer. Translated as Substance and function. Chicago: Open Court, 1923.

Cassirer, E. 1912/2005. Herman Cohen and the renewal of Kantian philosophy (trans. By Lydia Patton). Angelaki 10(1), 95-104.

Clifton, R. 1998. Scientific explanation in quantum theory. PhilSci Archive. <philsciarchive.pitt.edu/archive/ 0000009 1/>.

Colyvan, M. 2001. The indispensability of mathematics. Oxford: Oxford University Press. 
Coloyvan, M. 2002. Mathematics and aesthetic considerations in science. Mind 111(441), 69-74.

Daly, C. and Langford, S. 2009. Mathematical explanation and indispensability arguments. Philosophical Quarterly, 59(237), 641-658.

Dorato, M. and Felline, L. 2011. Scientific explanation and scientific structuralism. In A. Bokulich and P. Bokulich (eds.), Scientific structuralism, Boston Studies in the Philosophy of Science, Dordrecht: Springer, pp. 161-177.

du Bois-Reymond, P. 1875. Versuch einer classification der willkürlichen functionen reeller argumente nach ihren aenderungen in den kleinsten intervallen. Journal für die reine und angewandte Mathematik 79, 21-37.

Einstein, A. 1933/1954. On the method of theoretical physics. In A. Einstein, Ideas and opinions (new translations and revisions by S. Bargmann), New York: Bonanza Books (1954), pp. 270276.

Euler, L. 1736/1956. Solutio problematis ad geometriam situs pertinentis. Commentarii Academiae Scientarum Imperialis Petropolitanae 8, 128-140.

French, S. and Ladyman, J. 1998. A semantic perspective on idealization in quantum mechanics. In N. Shanks (ed.), Idealization in contemporary physics, Amsterdam: Rodopi, pp. 51-73.

Felline, L. 2018. Mechanisms meet structural explanations. Synthese 195(1), 99-114.

Ferreirós, J. 2017. Wigner's "unreasonable effectiveness" in context. Mathematical Intelligencer $39(2), 64-71$.

Feynman, R., et al. 1963. The Feynman lectures in physics, Vol. 2. Reading, Mass.: AddisonWesley.

Frigg, R. and Nguyen, J. 2018. Scientific representation. The Stanford Encyclopedia of philosophy (Winter 2018 Edition). In E. N. Zalta (ed.), URL= $<$ https://plato.stanford.edu/entries/scientific-representation/>.

Galilei, G. (1623/1960). The Assayer. In the controversy of the comets of 1618 (Trans., S. Drake). Philadelphia: The University of Pennsylvania Press.

Goldstein, S. 2017. Bohmian mechanics. The Stanford Encyclopedia of Philosophy (Summer 2017 Edition), E. N. Zalta (ed.). URL=<https://plato.stanford.edu/sum2017/entries/qm-bohm/>.

Hopkins, B. and Wilson, R. J. 2004. The truth about Königsberg. The College Mathematics Journal 35(3), 198-207.

Huggett, N. (ed.). 1999. Space from Zeno to Einstein: Classic readings with a contemporary commentary. Cambridge, MA: MIT Press.

Huggett, N. 2019. Zeno paradoxes. The Stanford Encylopedia of Philosophy (Spring 2019

Edition), E. N. Zalta (ed.). URL=

$<$ https://plato.stanford.edu/archives/spr2019/entries/paradox-zeno/>

Hughes, R. I. G. (1993). Theoretical explanation. Midwest Studies in Philosophy XVIII, 132-153.

Islami, A. 2017. A match not made in heaven: On the applicability of mathematics in physics. Synthese 194(12), 4839-4861.

Janssen, L. and Saatsi, J. (forthcoming). Explanatory abstraction. British Journal for the Philosophy of Science. 
Katz, V. J. 2009. A history of mathematics: An introduction ( $3^{\text {rd }}$ ed.). Addision-Wesley, Reading: Mass.

Koo, A. 2015. Mathematical explanation in science. PhD thesis, IHPST, University of Toronto.

Koslicki, K. 2012. Varieties of ontological dependence. In F. Correia and B. Schnieder (eds.), Metaphysical grounding: Understanding the structure of reality, Cambridge: Cambridge University Press, pp. 186-213.

Kowalewski, G. 1923. Über Bolzanos nichtdiffrenzierbare stetige funktion, Acta Mathematica 44, 315-319.

Lange, M. 2016. Because without cause: Non-causal explanations in science and mathematics. Oxford: Oxford University Press.

Lange, M. 2018. Reply to my critics: On explanations by constraint. Metascience 27(1), 27-36.

Leng, M. 2005. Mathematical explanation. In C. Cellucci and D. Gillies (eds.), Mathematical reasoning and heuristics. London: King's College Publishing, pp. 167-189.

Lévy-Leblond, J-M. 1992. Why does physics need mathematics? In E. Ullmann-Margalit (ed.), The scientific enterprise, Boston Studies in the Philosophy of Science vol. 146, Dordrecht: Springer, pp. 145-161.

Lützen, J. 2011. The physical origin of physically useful mathematics. Interdisciplinary Science Reviews 36(3), 229-243

Lyon, A. 2012. Mathematical explanations of empirical facts, and mathematical realism. Australasian Journal of Philosophy 90(3), 559-578.

Mancosu, P. 2018. Explanation in Mathematics. The Stanford Encyclopedia of Philosophy (Summer 2018 Edition), E. N. Zalta (ed.), URL= $<$ https://plato.stanford.edu/archives/sum2018/entries/mathematics-explanation/>

Mandelbrot, B. 1977. Fractals, form, chance and dimension. San Francisco: W.H. Freeman.

Melia, J. 2000. Weaseling away the indispensability argument. Mind 109(435), 455-479.

Melia, J. 2002. Response to Colyvan. Mind 111(441), 75-79.

Mundy, B. 1986. On the general theory of meaningful representation. Synthese 67(3), 391-437.

Neuenschwander, E. 1978. Riemann's example of a continuous "nondifferentiable" function. Mathematical Intelligencer 1, 40-44.

Pincock, C. 2004. A new perspective on the problem of applying mathematics. Philosophia Mathematica 12(2), 135-161.

Pincock, C. 2011a. Abstract explanation and difference making. A colloquium presentation in the Munich Center for Mathematical Philosophy, LMU, December 12, 2011.

Pincock, C. 2011b. Discussion note: Batterman's "On the explanatory role of mathematics in empirical science". British Journal for the Philosophy of Science 62(1), 211-17.

Pincock, C. 2012. Mathematics and scientific representation. Oxford: Oxford University Press.

Pincock, C. 2015. Abstract explanations in science. British Journal for the Philosophy of Science 66(4), 857-882. 
Pitowsky, I. 1992. Why does physics need mathematics? A comment. In E. Ullmann-Margalit (ed.), The scientific enterprise, Boston Studies in the Philosophy of Science vol. 146, Dordrecht: Springer, pp. 163-167.

Poincare, H., 1913. La valeur de la science. Geneva: Le Cheval Aile.

Redhead, M. 2001. Quests of a realist: Review of Stathis Psillos's Scientific realism: How science tracks truth, Metascience 10(3), 341-347.

Resnik, M. D. 1997. Mathematics as a science of patterns. Oxford: Clarendon Press.

Saatsi, J. 2011. The enhanced indispensability argument: Representational versus explanatory role of mathematics in science. British Journal for the Philosophy of Science 62(1), 143-54.

Saatsi, J. 2016. On the "Indispensable explanatory role" of mathematics. Mind 125(500), 10451070.

Saatsi, J. 2018. A pluralist account of non-causal explanations in science and mathematics: Review of M. Lange's Because without cause: Non-causal explanation in science and mathematics. Metascience 27(1), 3-9.

Shapiro, S. (1997), Philosophy of mathematics: Structure and ontology. Oxford: Oxford University Press.

Steiner, M. 1978. Mathematical explanations. Philosophical Studies 34(2), 135-151.

Steiner, M. 1998. The applicability of mathematics as a philosophical problem. Cambridge, MA: Harvard University Press.

Stemeroff, N. 2018. Mathematics, structuralism, and the promise of realism: A study of the ontological and epistemological implications of mathematical representation in the physical sciences. A PhD thesis submitted to the University of Toronto.

Strevens, M. 2008. Depth: An account of scientific explanation. Cambridge, MA: Harvard University Press.

Vineberg, S. (2018). Mathematical explanation and indispensability. Theoria 33(2), 233-247.

Weierstrass, K. 1895. Über continuirliche functionen eines reellen arguments, die für keinen werth des letzteren einen bestimmten differentialquotienten besitzen (read 1872). In Mathematische werke von Karl Weierstrass, Vol. 2, p. 71-76, Berlin 1895

Wiener, N. 1923. Differential space. Journal Mathematical Physics 2, 131-174.

Wigner, E. 1960. The unreasonable effectiveness of mathematics in the natural sciences. Communications in Pure and Applied Mathematics 13(1), 1-14.

Woodward, J. 2003. Making things happen. Oxford: Oxford University Press. 